\title{
Effects of Turbulence on Mass Transfer of Cylindrical Disks
}

\author{
Herricos Stapountzis, Georgios Andreatos, Ioannis Kordatos \\ University of Thessaly, Department of Mechanical Engineering \\ Leoforos Athinon, Pedion Areos, Volos 383 34, Greece \\ erikos@uth.gr, andreato.g512@gmail.com,giannhskordatos@hotmail.com
}

\begin{abstract}
Mass transfer from solid cylindrical disks of Camphor has been studied in a wind tunnel, in a synthetic jet turbulence chamber with zero mean speed and in an axisymmetric turbulent jet. Mass decay rates were measured by monitoring the shape of the models . Average Sherwood numbers were computed and their dependence on turbulent intensity was examined. It was found that turbulent intensity increased the rate of mass transfer.
\end{abstract}

Keywords: Mass transfer, Camphor sublimation, grid turbulence, homogeneous and isotropic turbulence, synthetic jets.

\section{Introduction}

There is considerable interest in the study of the rate of mass transfer from or to surfaces because of the direct applications to many chemical engineering unit operations, but also because it lends itself as an alternative method to obtain convective heat transfer coefficients. The mass transfer results can be converted to heat transfer results by a heat / mass transfer analogy because of the similarity of the governing equations between heat and mass transfer [1], [2]. Sublimation is one of the most convenient processes that can be used to study mass transfer. In this method the surface under consideration is coated with a feebly volatile substance which can sublimate like Naphthalene or Camphor. The greatest rates of sublimation are usually achieved in regions of high shear stress or skin friction. Boundary conditions that are approximate or difficult to attain in heat transfer experiments like adiabatic or isothermal boundary conditions, are readily applicable in the mass transfer counterpart. For example surfaces coated or consisting of Camphor correspond to the isothermal wall condition (then the Camphor vapour pressure and concentration at the surface would be uniform uniform) and non-subliming surfaces are equivalent to adiabatic wall conditions. Due to diffusion and convection the mass loss of Camphor occurs continuously on the surface. Temperature gradients and heat flux correspond to concentration gradients and mass flux. Measurement of the sublimation depth would yield local mass transfer rates. The aforementioned advantages promoted research in different flow regimes (Mach numbers $>1$ or $<1$ ), various body shapes (airfoils , circular cylinders, disks, spheres, prolate spheroids, cones etc), [3], [4], [5], [6] and flow conditions (turbulent, non-turbulent flows), [7], [8]. There is still some controversy concerning the effects of free stream turbulence level and the length scale on the average Sherwood number on disk shapes. To this end, an experimental investigation was carried out and presented in this paper, i.e. study the effect of turbulence on the Sherwood numbers of disk shapes, by employing the Camphor sublimation technique.

\section{Experimental Setup and Procedures}

Since the main objective of the present study was to investigate the influence of turbulent flow on mass transfer from circular disks, three types of flows were employed: Turbulent flow generated by a grid in a wind tunnel, turbulent flow generated by synthetic jets in a "turbulence chamber" and the turbulent flow of an axisymmetric circular jet. Mass transfer was measured by the method of sublimation. Camphor was opted against Naphthalene for health reasons, because appropriate ventilation conditions were not available.

\subsection{Experimental Models}

A single type of test model for sublimation has been used for all flow configurations. That is, a short circular cylinder in the form of a disk (tablet) with initial base diameter $\mathrm{D}_{0}=25.3 \mathrm{~mm}$ and height $\mathrm{H}_{0}=4.2 \mathrm{~mm}$. The material of the solid disk was commercially available Camphor, sold in many stores for domestic use as moth repellent. This selection 
was dictated by the need to keep a large number of identical tokens for all experiments, avoiding machining or complicated casting procedures, [1]. The disadvantages are those of fixed geometry and uncertainty about the consistency in composition and chemical properties. In this study it has been assumed that the Camphor employed for the experiments had properties similar to those for Laboratory use, thus allowing to draw data from the reference books like the Handbook of Chemistry and Physics [9]. The density $\rho_{\mathrm{C}}$ was estimated by weighing the disk and dividing by its volume. The result was $m_{0}=0.0021 \mathrm{~kg}$ and $\rho_{\mathrm{C}}=992 \mathrm{~kg} / \mathrm{m}^{3}$. Molecular Weight, $\mathrm{MW}_{\mathrm{c}}$, was taken equal to $152.24 \mathrm{gr} / \mathrm{mol}$ and the Schmidt number $\mathrm{Sc}=v / \mathrm{D}_{\mathrm{c}}=3.2258$ over a wide range of temperatures, since the kinematic viscosity, $v$, of air and the mass diffusion coefficient of the Camphor vapor, $\mathrm{D}_{\mathrm{c}}$, vary in a similar manner with temperature.

The models were supported by a thin plastic threaded rod in the center and secured by a small nut, the circular base facing the flow. It is recognized that this method of support should influence the flow conditions and subsequently the sublimation rate in the region of the front stagnation point. However, it was preferred to the other tried method of back support with a set of needles, because in that case the model was shaking in strong turbulent flow conditions.

\subsection{Generation of Turbulent Flows}

The first type of flow was that of Grid Turbulence in a wind tunnel. The wind tunnel was of the suction type with test section $0.52 \mathrm{X} 0.72 \mathrm{~m}$ (width $\mathrm{X}$ height) and length of working section $2.7 \mathrm{~m}$. With a biplanar grid of square mesh size $\mathrm{M}=90 \mathrm{~mm}$ and due to the limitations of the tunnel motor, the maximum attainable streamwise speed was $\mathrm{U}_{0}=12 \mathrm{~m} / \mathrm{s}$ and the Reynolds number based on the initial model diameter was $\operatorname{Re}_{\mathrm{D} 0}=2 \times 10^{4}$. Tests were carried out only at this Re number, but the effect of turbulence was examined from the point of view of changing the turbulence intensity $i=u_{R M S} / U_{0}$. This was accomplished by positioning the model at various streamwise locations $\mathrm{x}$ along the centreline of the tunnel. Mean and fluctuating velocities were measured with Pitot-static tubes and hot wires. It is known, [10] , that the intensity i decays with distance from the grid, while the turbulence length scale $\mathrm{L}_{\mathrm{xu}}$ increases, which means that the turbulence is nearly homogeneous and isotropic in the transverse direction, but inhomogeneous in the $\mathrm{x}$ direction. For constant Re number, the mass transfer is expected to be influenced by changes in both the intensity and the turbulence length scale necessitating the use of many grids. For comparison reasons, sublimation tests were made with uniform flow, without a grid. Turbulence data for the wind tunnel experiments are summarized in Table 1.

The second type of turbulent flow was that produced in a "turbulence chamber" of Synthetic Jets [11], [12]. The advantage of this flow configuration is that the mean flow is nearly zero, $\mathrm{U}_{0} \approx 0$, while the turbulent intensity can be changed by conveniently varying the Synthetic Jet excitation. Therefore, it is attempted to isolate the influence of turbulence intensity since the effect of convection by $\mathrm{U}_{0}$ would be absent. In the central region of the turbulence chamber the flow is nearly Homogeneous and Isotropic. The radius of this nearly spherical region is $\mathrm{R}=150 \mathrm{~mm} \gg \mathrm{D}_{0}$. It is also possible to heat up the flow by inserting electrical resistances in the Synthetic Jets. Flow data, obtained by LDA and Constant Current Anemometer for the temperature fluctuations are shown in Table 1.

The third type of flow employed was the turbulent flow downstream of an axisymmetric Circular Jet. The model was positioned along the jet centreline at a streamwise location, $\mathrm{x}_{\mathrm{J}}$, where the mean centerline speed $\mathrm{U}_{\mathrm{c}}$ equals that of the wind tunnel i.e. $U_{c}=U_{0}=12 \mathrm{~m} / \mathrm{s}$. The flow there is highly anisotropic and, for the present setup the turbulent intensity is larger than that of the wind tunnel as well as the length scale $\mathrm{L}_{\mathrm{xu}}[13]$.

Table 1: Flow data for wind tunnel, turbulence chamber and circular jet.

\begin{tabular}{|c|c|c|c|c|c|c|}
\hline Flow type & $\mathrm{U}_{0}, \mathrm{~m} / \mathrm{s}$ & $\mathrm{Uc} \mathrm{m} / \mathrm{s}$ & $\mathrm{x} / \mathrm{M}=10$ & $\mathrm{x} / \mathrm{M}=15$ & $\mathrm{x} / \mathrm{M}=20$ & $\mathrm{x} / \mathrm{M}=25$ \\
\hline $\begin{array}{l}\text { Grid } \\
\text { Turbulence }\end{array}$ & $12 \mathrm{~m} / \mathrm{s}$ & & $\begin{array}{l}\mathrm{i}=0.071 \\
\mathrm{~L}_{\mathrm{xu}} / \mathrm{D}_{0} \approx 1.20\end{array}$ & $\begin{array}{l}\mathrm{i}=0.056 \\
\mathrm{~L}_{\mathrm{xu}} / \mathrm{D}_{0} \approx 1.46\end{array}$ & $\begin{array}{l}\mathrm{i}=0.048 \\
\mathrm{~L}_{\mathrm{xu}} / \mathrm{D}_{0} \approx 1.67\end{array}$ & $\begin{array}{l}\mathrm{i}=0.045 \\
\mathrm{~L}_{\mathrm{xu}} / \mathrm{D}_{0} \approx 1.78\end{array}$ \\
\hline $\begin{array}{l}\text { Synthetic } \\
\text { Jets }\end{array}$ & $\mathrm{U}_{0} \approx 0$ & & $\begin{array}{l}\text { "HOT" } \\
\mathrm{U}_{\mathrm{RMS}}=0.47 \mathrm{~m} / \mathrm{s}, \\
\Theta=40{ }^{\circ} \mathrm{C} \\
\Theta_{\mathrm{RMS}}=2.5^{0} \mathrm{C}\end{array}$ & $\begin{array}{l}\text { "WARM" } \\
\mathrm{U}_{\mathrm{RMS}}=0.26 \mathrm{~m} / \mathrm{s}, \\
\Theta=32{ }^{\circ} \mathrm{C} \\
\Theta_{\mathrm{RMS}}=1.6^{\circ} \mathrm{C}\end{array}$ & $\begin{array}{l}\text { "COLD" } \\
\mathrm{U}_{\mathrm{RMS}}=0.79 \mathrm{~m} / \mathrm{s}, \\
\Theta=22{ }^{\circ} \mathrm{C}\end{array}$ & \\
\hline Circular Jet & & $12 \mathrm{~m} / \mathrm{s}$ & $\mathrm{x} / \mathrm{D}_{\mathrm{J}}=25$ & $\mathrm{~L}_{\mathrm{xu}} / \mathrm{D}_{0}$ & & \\
\hline
\end{tabular}




\subsection{Measurement Method for Sublimation}

The mass transfer rate is inferred from the loss of mass due to sublimation either on the average or locally. In the present work the former is pursued. Weighing the specimens over prescribed time intervals is the most frequent method. It has been tried in this work, but due to the small size of the models, errors were introduced by handling the Camphor disk and affecting its shape ; also by taking it out and putting it back again sometimes has caused the model to break or be misaligned with respect to its previous position. An alternative method has been followed, that of monitoring the evolution of its shape and size with time by taking still pictures and videos from different viewing points (top, front, back sides). After digital image processing the dimensions of the model could be computed and consequently its volume and remaining mass. Comparison with the weighing method gave good agreement. The most trustworthy data were those obtained at current times before the model started being deformed asymmetrically. Also, when the model shrinks to a very small size, the flow conditions would change significantly due to the effect of the supporting threaded rod and the securing nut.

\section{Experimental Results and Discussion}

\subsection{Computation of the Mass Transfer Coefficients and Sherwood Numbers}

There are analytical expressions for the sublimation due to natural convection of various shapes (sphere, cylinder, [14]) Here the average mass transfer coefficient $h_{m}$ will be computed from the measured mass loss and the molecular properties of the fluids involved i.e. air and Camphor vapour [15]. The molecular properties of the fluids are affected by temperature as also the process of sublimation as whole, as shown in Fig. 1. Sublimation was monitored in a heated air stream of $\mathrm{U}_{0}=5.5 \mathrm{~m} / \mathrm{s}$ and low turbulence intensity, and it was found that the rate of mass sublimation, $\mathrm{dm} / \mathrm{dt}$, was a strong function of the air temperature, $\mathrm{dm} / \mathrm{dt} \approx 3.85 \Theta^{2.5}$.

Fig. 1: Effect of air temperature on rate of mass sublimation.

The mass transfer coefficient $\mathrm{h}_{\mathrm{m}}$ is defined here as

$$
\mathrm{h}_{\mathrm{m}}=\frac{d m}{d t} \frac{1}{\rho A\left(w_{c s}-w_{c \infty}\right)} \quad[\mathrm{m} / \mathrm{s}]
$$

where $\rho$ is the air density, A is the area over which sublimation occurs. A is taken here equal to the initial total surface area of the disk $\left(=\pi \mathrm{D}_{0}^{2} / 4 \times 2+\pi \mathrm{D}_{0} \mathrm{H}_{0}=0.001339 \mathrm{~m}^{2}\right)$. This includes the front and back surfaces where flow conditions are different, but it is thought that this is a first step in calculating the average mass transfer. $\mathrm{w}_{\mathrm{cs}}, \mathrm{w}_{\mathrm{c \infty}} \operatorname{are}_{\mathrm{s}}$ the mass fractions of Camphor vapor at the model surface and at infinity respectively. It is assumed that $\mathrm{w}_{\mathrm{c} \infty}$ would be zero if the flowing medium is free of Camphor vapor, an assumption reasonable for the wind tunnel and jet cases. This assumption might be criticized in the synthetic jet case, if due to intense sublimation, the Camphor concentration in the 
surrounding air is raised to very high levels. In the turbulence chamber the turbulent mixing is very strong, therefore it is realistic to accept the validity of this assumption, $\mathrm{w}_{\mathrm{c} \infty}=0$, also for the synthetic jet turbulent flow. The mass fraction $\mathrm{w}_{\mathrm{cs}}$ on the surface depends on the Camphor vapor pressure $\mathrm{P}_{\mathrm{c}}$, the air pressure $\mathrm{P}_{\mathrm{a}}$ (atmospheric) and the Molecular Weights of Camphor vapor, $\mathrm{MW}_{\mathrm{c}}$ and air $\mathrm{MW}_{\mathrm{a}}$.

$$
W_{C S}=\frac{P_{c}}{P_{a}} \frac{M W_{c}}{M C_{a}}=\frac{P_{c}}{101325} \frac{152.24 \mathrm{~kg} / \mathrm{mol}}{29 \mathrm{~kg} / \mathrm{mol}} \quad[-]
$$

For each run, the temperature near the sublimating surface is recorded and the Camphor vapor pressure $\mathrm{P}_{c}$ is computed. Thereafter, the Sherwood number, Sh, is computed from the equation.

$$
\mathrm{Sh}=h_{m} \frac{D_{0}}{D_{c}} \quad[-]
$$

\subsection{Mass Transfer in Grid Turbulence}

The amount of sublimated Camphor mass, $\mathrm{m}_{0}-\mathrm{m}(\mathrm{t})$ in the wind tunnel experiments with Grid Turbulence is shown in Fig. 2 nondimenionalized by the initial mass. The wind tunnel speed $U_{0}$ was $12 \mathrm{~m} / \mathrm{s}$. The turbulence Reynolds number based on the length scale $\mathrm{L}_{\mathrm{xu}}$ and the $\mathrm{u}$ velocity fluctuation $\mathrm{u}_{\mathrm{RMS}}$, varies from 1724 in the beginning, $\mathrm{x} / \mathrm{M}=10$ to 1621 at the last downstream location, $\mathrm{x} / \mathrm{M}=25$ indicating the existence of a strong inertial subrange. Turbulence therefore is expected to play a significant role in the mass transfer and this is depicted in the results for $\mathrm{dm} / \mathrm{dt}$. The mass decay rate is almost halved when the turbulence intensity drops from 0.071 to 0.045 . However the role of the increasing length should also be taken into account, since it is known to cause a decrease in the mass transfer. Sublimation in a nominally "smooth" flow without the grid resulted in a considerably lower rate of $2.12 \times 10^{-8} \mathrm{~kg} / \mathrm{s}$. The intensity in this case was 0.007 . It is also observed that the value of the sublimated mass varies almost linearly with time, suggesting that the shrinking of the disk size does not alter the flow around it significantly. The computation of the decay rate was based on data in the initial stages of sublimation, when the disk shape maintained its symmetry.

Fig. 2: Mass transfer in the wind tunnel experiments with turbulence producing grid at locations $x / M=10,15,20,25$ and intensities $\mathrm{i}=0.071,0.056,0.048$ and 0.045 respectively. $\mathrm{U}_{0}=12 \mathrm{~m} / \mathrm{s}$. 


\subsection{Mass Transfer in the Turbulence Chamber and the Axisymmetric Jet}

The sublimation in the synthetic jet facility and the axisymmetric jet is shown in Fig. 3. The case with high heating $\left(40{ }^{0} \mathrm{C}\right)$ exhibits the greater mass transfer rate. However, as will be shown later, the resulting Sherwood number does not follow the same trend because of the counteracting effect of the mass diffusion coefficient $\mathrm{D}_{\mathrm{c}}$. Besides having different $\mathrm{u}_{\mathrm{RMS}}$ velocities, the synthetic jets have also different levels of fluctuating temperatures $\theta_{\mathrm{RMS}}$, a fact that it is also expected to influence the rate of sublimation. It is noticed that the synthetic jets that are not heated $\left(22{ }^{0} \mathrm{C}\right)$ but have the highest level of fluctuating velocities $\left(\mathrm{u}_{\mathrm{RMS}}=0.79 \mathrm{~m} / \mathrm{s}\right.$ ) lead to values of $\mathrm{dm} / \mathrm{dt}$ comparable to those in the wind tunnel for similar $\mathrm{u}_{\mathrm{RMS}}$ $(0.85 \mathrm{~m} / \mathrm{s})$, despite the fact that the mean velocity $\mathrm{U}_{0}$ in the synthetic jet facility is zero. It seems therefore that turbulence fluctuations round the model are able to cause significant mass transport, that is turbulence enhances the sublimation process. On the other hand, the axisymmetric jet with its largest $u_{\text {RMS }}$ (Table 1) does not result in exceedingly big mass rates, presented in Fig. 3. It is known that along with large velocity fluctuations, the single jet has transverse fluctuating velocity components $\mathrm{v}, \mathrm{w}$ of smaller magnitude due to its anisotropic nature, but also considerably larger length scales, which tend to act against mass transfer. In Fig. 3 it is also shown the mass transfer due to natural convection, almost two orders of magnitude smaller than the synthetic jet values. Mass transfer due to natural convection and in the synthetic jet facility share a common feature, that the mean velocity round the model is zero, however mass transport is greatly affected by the nearly isotropic and homogeneous turbulent velocity fluctuations.

Fig. 3: Mass transfer in the turbulence chamber of synthetic jets and the axisymmetric jet with $\operatorname{Re}_{\mathrm{D} 0}=20000$.

\subsection{Sherwood Numbers}

Using equation (3) and taking into account the appropriate values of the mass diffusion coefficients for Camphor vapour, the Sherwood numbers, Sh, were computed for all cases and the results are shown in Fig. 4. The strong influence of the turbulence fluctuating velocity is demonstrated for both the grid turbulence and the synthetic jet turbulence. As presented here, the data do not collapse into a single curve, at least for the grid turbulence and the axisymmetric jet, where the Reynolds numbers for the model are the same $(=20000)$, since the length scale of turbulence, anisotropy and other factors might affect the mass transfer. In this study, there no sufficient data that would allow to take into consideration these effects. The data available in the literature refer to different geometrical shapes like circular cylinders, flat plates, airfoils and spheres and mainly to local and not average Sh numbers. For example in [7] it was found that the Sherwood number at the front stagnation of a circular cylinder increased by $60 \%$ when the free stream turbulence changed from 0.2 
$\%$ to $23 \%$. When a disk is examined, usually mass transfer is limited only to the frontal area, while in the present work mass transfer takes place over the whole model area. Such data for smooth flow and $\mathrm{Re}=20000$ are shown in Fig. 4 for disks facing the flow but allowing mass transfer only in the frontal side, [3], [5], [6]. Their results are in qualitative agreement with our results for the wind tunnel tests and no grid.

An attempt was made to use existing correlations for different shapes that take into account the effect of turbulence length scale and the turbulence intensity. In Fig. 5 the parameter $\lambda$ is used which, according to [16], could make the data for the normalized Sherwood number $\mathrm{Sh} / \mathrm{Sh}_{0}$ to collapse into a single curve for sublimation of naphthalene from circular cylinders in grid turbulence. This parameter is given as

$$
\lambda=1+131.05 \mathrm{i}^{0.317} \operatorname{Re}^{-0.492}\left(\mathrm{~L}_{\mathrm{xu}} / \mathrm{D}_{0}\right)^{-0.137} \quad[-]
$$

Implying that $\mathrm{Sh}_{0}$ is the value of the Sherwood number for nearly zero turbulence intensity. We have used the results for grid turbulence of our experiments, where $\mathrm{Sh}_{0}$ was the value for no grid $(\mathrm{i} \approx 0.007)$. The data do not extend to a sufficient range in order to draw a definite conclusion.

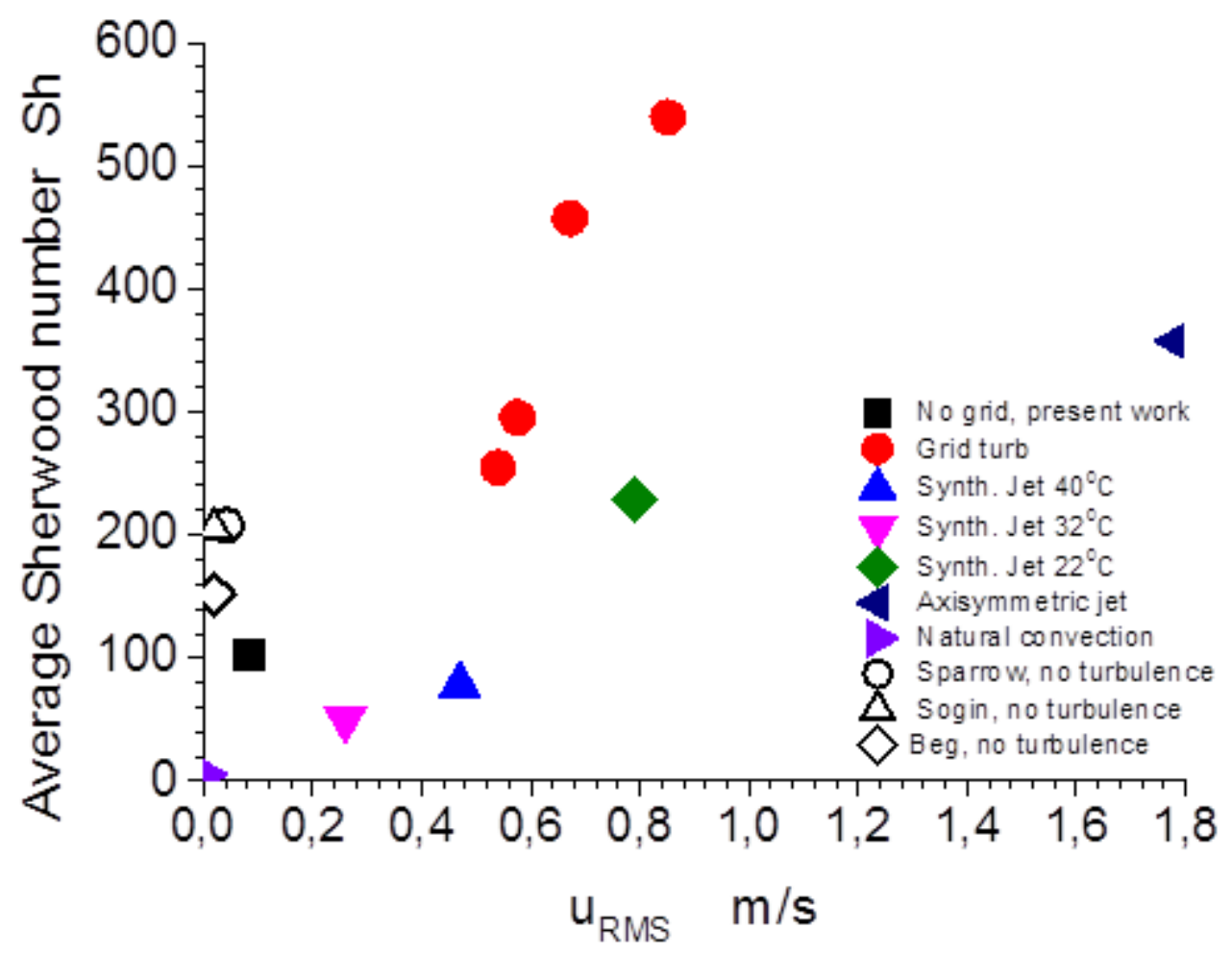

Fig. 4: Sherwood numbers for disks and different flow configurations, $\mathrm{Re}_{\mathrm{D} 0}=20000$, present results and those from [3], [5] and [6].

\section{Conclusions}

Sublimation experiments with solid Camphor disks exposed to different types of turbulent flows showed that turbulent velocity fluctuations are the prevailing factor for the enhancement of the average mass transfer. Grid turbulence in a wind tunnel may increase Sherwood numbers by almost an order of magnitude with respect to the corresponding values in uniform flow at the same Reynolds number. Even if there is no mean flow, $\mathrm{U}_{0}=0$, turbulent velocity fluctuations act as to increase the Sherwood number. There is some evidence that increased turbulence length scales would tend to affect mass transport in the opposite direction. This is in line with findings for the effect of turbulence length scale on the heat transfer at the front stagnation point of several body shapes.

For the solid disks considered in the present investigation, the mass varies linearly with time at least over periods when almost a third of the total mass has been sublimated. 
At constant heated air flow and low turbulence level, the sublimation mass rate goes as temperature to the power of 2.5, the reduction of volume, from which the reduction of mass was deduced, was computed by taking snapshots of the model at prescribed times and then processing them digitally. There was good agreement with the weighing method provided the model has not been significantly distorted by the turbulent flow.

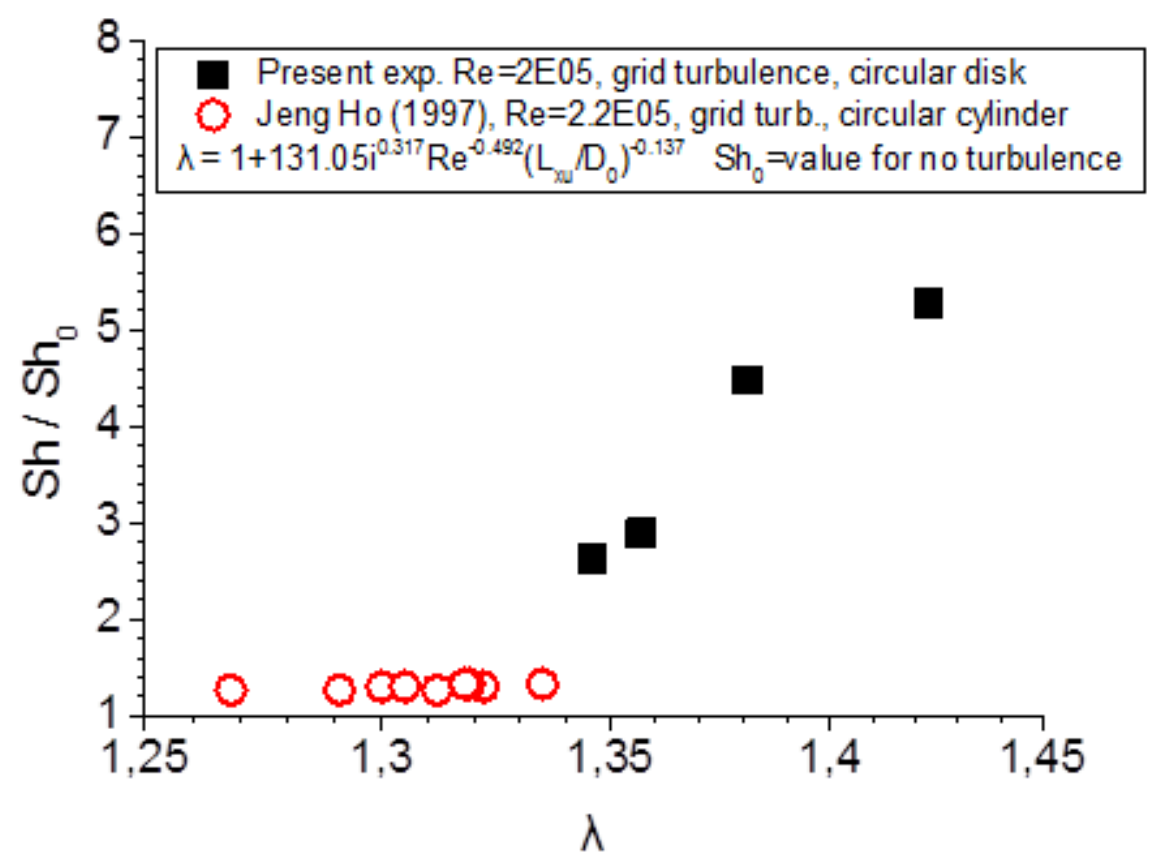

Fig. 5: Contribution of turbulence intensity and length scale on the normalized Sherwood numbers for circular cylinders, [16], and present work.

\section{Acknowledgements}

Special thanks to Drs Georgios Charalampous and Dimitrios Tziourtzioumis for their assistance in the LDA measurements.

\section{References}

[1] R. J. Goldstein and H. H. Cho, "A review of mass transfer measurements using Naphthalene sublimation," Exp. Thermal and Fluid Sci., vol. 10, pp. 416-434, 1995.

[2] P. R. Souza Mendes, "The Naphthalene sublimation technique," Exp. Thermal and Fluid Sci., vol. 4, pp. 510-523, 1991.

[3] E. M. Sparrow and G. T. Geiger, "Local and average transfer characteristics for a disk situated perpendicular to a uniform flow," Journ. Heat Transfer, vol. 107, pp. 321-326, 1985.

[4] H. H. Sogin, "Sublimation from disks to air streams flowing normal to theirn surfaces," ASME Trans. J. Heat Transfer, vol. 80, pp. 61-71, 1958.

[5] H. H. Sogin, "An improved correlation of stagnation point mass transfer from Naphthalene circular disks facing uniform air streams," ASME Trans. J. Heat Transfer, vol. 113, pp. 772-773, 1991.

[6] S. A. Beg, "Forced convective mass transfer from circular disks," Warme und Stoffubertragung, vol. 6, pp. 45-51, 1973.

[7] S. Sanitjai and R. J. Goldstein, "Effect of free stream turbulence on local mass transfer from a circular cylinder," Int. J. Heat Mass Transfer, vol. 44, pp. 2863-2875, 2001.

[8] G. J. Van Fossen, R. J. Simoneau, and C. Y. Ching, "Influence of turbulence parameters, Re number and body shape on stagnation heat transfer," ASME Trans. J. Heat Transfer, vol. 117, pp. 597-603, 1995.

[9] D. F. Lide, Handbook of Chemistry and Physics, $74^{\text {th }}$ edition. CRC, pp. 3-158, 6-87, 1993-1994. 
[10] H. Stapountzis, B. L. Sawford, J. C. R. Hunt, and R. E. Britter, "Structure of the temperature field downwind of a line source in grid turbulence," Journ. Fl. Mech., vol. 186, pp. 401-424, 1986.

[11] H. Stapountzis H. G. Charalampous, D. Tziourtzioumis, and A. Stamatelos, "Diffusion in synthetic jet generated turbulence," in 4th International Conference on Jets, Wakes and Separated Flows, ICJWSF2013, Nagoya, Japan, 2013.

[12] H. Stapountzis, N. Tsandouklas, and T. Demetriades, "Effects of Synthetic Jet Turbulence on Heated Line Plumes," in Proceedings of the International Conference on Heat Transfer and Fluid Flow, Prague, Czech Republic, August 11$12,2014$.

[13] I. Wygnanski and H. Fiedler, "Some measurements in the self-preserving jet," Journ. Fl. Mech., vol. 38, pp. 577-612, 1969.

[14] B. K. Dutta, Principles of mass transfer and separation processes. New Dehli: PHI Learning, 2009.

[15] Y. A Cengel and A. J. Ghajar, Heat and Mass Transfer - Fundamentals and Applications, 4th Edition. New York, NY: McGraw-Hill, 2010.

[16] C-J. Ho, "Effect of length scale on heat transfer from cylinder in cross flow," M.Sc. Thesis, Dept. Mech. Eng., Texas Tech. Univ., 1979. 